Nota técnica

Volumen 33(1): Artículo 44555, 2022

e-ISSN 2215-3608, doi:10.15517/am.v33i1.44555

https://revistas.ucr.ac.cr/index.php/agromeso/index

\title{
Propiedades químicas del aceite de cinco genotipos de Jatropha curcas L., en Colombia $^{1}$
}

\section{Chemical properties of the oil of five genotypes of Jatropha curcas L., in Colombia}

\author{
Luis Fernando Campuzano-Duque ${ }^{2}$,Fernando Cardeño-López ${ }^{3}$
}

1 Recepción: 16 de noviembre, 2020. Aceptación: 16 de mayo, 2021. Este trabajo formó parte del Proyecto Plataforma Jatropha Colombia, financiado por el Ministerio de Agricultura y Desarrollo Rural (MADR); Empresa Colombiana de Petróleo (Ecopetrol), Carbones de Colombia (Cerrejón) y Departamento Nacional de Ciencia y Tecnología (Colciencias).

2 Corporación Colombiana de Investigación Agropecuaria (AGROSAVIA). Centro de Investigación La Libertad. Villavicencio, Meta, Colombia. lcampuzano@agrosavia.co (autor para correspondencia; https://orcid.org/0000-0003-1146-4591).

3 Universidad de Antioquia (UdeA). Medellín, Facultad de Ingeniería, Grupo Procesos Químico-Industriales. Antioquia, Colombia. fernando. cardeno@udea.edu.co (https://orcid.org/0000-0001-8232-3505).

\section{Resumen}

Introducción. Jatropha curcas L. (JC), es una especie multipropósito con aplicaciones biológicas, farmacológicas e industriales. Su aceite es reconocido por la composición dominante de los ácidos grasos oleico-linoleico que lo hacen apto como biodiésel en motores de combustión interna, sin hacer cambios importantes al diseño del motor. Objetivo. Identificar en el aceite de cinco genotipos de JC en Colombia los índices de cetano, peróxido, yodo, el valor de acidez, el poder calorífico y verificar si cumplen con las normas para producción de biodiésel. Materiales y métodos. Se realizó un experimento en Espinal, Colombia, en un diseño de bloques completos al azar con cinco tratamientos y tres repeticiones. Los tratamientos estuvieron constituidos por cinco genotipos de JC. Se utilizó el fruto de la quinta cosecha en el año 2015. Del aceite se determinaron cinco ácidos grasos (oleico, linoleico, linolénico, palmítico y esteárico) y cinco factores químicos (índices de cetano, peróxido y yodo, acidez y poder calorífico). Resultados. El aceite de los cinco genotipos de JC evaluados en Colombia presentaron un perfil lipídico con predominancia de los ácidos grasos monoinsaturados (oleico C18:1) y poliinsaturados (linoleico C18:2). Los genotipos de JC evaluados en Colombia presentaron bajos índices de cetano, peróxido y yodo, baja acidez y alto poder calorífico. Conclusiones. Las propiedades de índices de cetano y yodo en los genotipos evaluados, cumplieron con las especificaciones de las normas ASTM (American Society for Testing and Materials) y EN (European Norm) para la producción de biodiésel, por lo que se catalogan como materia prima apropiada para la producción de este biocombustible.

Palabras clave: biodiésel, índice de cetano, acidez, índice de peróxido, índice de yodo, poder calorífico, perfil lipídico.

\section{Abstract}

Introduction. Jatropha curcas L. (JC) is a multipurpose species with biological, pharmacological, and industrial applications. Its oil is recognized by the dominant composition of oleic-linoleic fatty acids that make it suitable as 
biofuel in internal combustion engines, without making major changes to the engine design. Objective. To identify in the oil of five JC genotypes in Colombia the cetane, peroxide, iodine, the acidity value, the calorific value indixes and to verify if they comply with standards for biodiesel production. Materials and methods. An experiment was carried out in Espinal, Colombia, in a randomized complete block design with five treatments and three repetitions. The treatments consisted of five JC genotypes. The fruit from the fifth harvest in 2015 was used. Five fatty acids (oleic, linoleic, linolenic, palmitic, and stearic) and five chemical properties (cetane index, acidity, peroxide, iodine, and calorific value) were determined from the oil. Results. The oil from the five JC genotypes evaluated in Colombia presented a lipid profile with a predominance of monounsaturated (oleic C18: 1) and polyunsaturated (linoleic C18: 2) fatty acids. The JC genotypes evaluated in Colombia presented a low cetane, peroxide, and iodine indexes, low acidity, and high calorific value. Conclusions. The properties of cetane and iodine indexes, of the genotypes evaluated complied with the specifications of the American Society for Testing and Materials (ASTM) and the European Norm for biofuel production, so they are classified as appropriate raw material for biofuel production.

Keywords: biofuel, cetane number, acidity, peroxide index, iodine index, calorific value, lipid profile.

\section{Introducción}

El agotamiento gradual de los combustibles de origen fósil, indica que para cubrir la demanda energética a nivel mundial será necesario el uso de otras energías alternativas renovables con la ventaja de una reducción sustancial de la contaminación ambiental y el calentamiento global (Abobatta, 2019; Achten et al., 2008; Soliman \& He, 2015). La calidad de un aceite se determina por las propiedades químicas y físicas; sin embargo, las propiedades químicas basadas en el perfil de ácidos grasos y algunos índices químicos (cetano, acidez, peróxido y yodo) son las más importantes (Abobatta, 2019).

Cada especie vegetal tiene uno o dos ácidos grasos específicos dominantes, por ejemplo: la palma africana (Elaeis guineensis) está asociada con el ácido palmítico, la higuerilla (Ricinus communis) con el ricinoleico, el coco (Cocos nucífera) con el láurico, la oliva (Olea europaea), cacahuate (Arachis hypogaea) y el sésamo (Sesamum indicum) con el oleico, la colza (Brassica napus) con el behénico y eurícico, el cacao (Theobroma cacao) con el esteárico y palmitoleico, el algodón (Gossypium hirsutum), el cártamo (Carthamus tinctorius) y el lino (Linum usitatissimum) con el linoleico y la Jatropha (Jatropha curcas) con el oleico y linoleico. No obstante, la composición de estos en cada especie puede variar según el genotipo, las condiciones de clima y el manejo agronómico como la nutrición y el riego (Benatti et al., 2004).

La composición de los ácidos grasos, longitud de la cadena de carbono y el grado de saturación e insaturación, además de modular las propiedades del biodiésel, determinan algunas propiedades químicas del aceite como los índices de cetano, peróxido y yodo, y el valor de acidez (García-Dávila et al., 2014), las cuales repercuten en las propiedades del biodiésel, en los procesos de la transesterificación, en el funcionamiento de los motores diésel y en el almacenamiento del producto (Aminul et al., 2012; Zhu et al., 2016).

El aceite de palma africana (Elaeis guineensis) es el referente de los biocombustibles de primera generación, por su alta productividad de aceite ( $2 \mathrm{t} \mathrm{ha}^{-1}$ año $^{-1}$ ) (Rincón \& Martínez, 2009); no obstante, este presenta contenidos bajos de ácido linoleico, bajo índice de cetano y acidez baja, que repercuten en un bajo desempeño en propiedades de flujo en frío (Jain \& Sharma, 2011).

Jatropha curcas (JC), además de presentar la ventaja de la composición de sus ácidos grasos que lo hacen apto como combustible, tiene fácil adaptación en áreas con suelos con limitaciones nutricionales y baja precipitación, lo cual le da más ventajas sobre aceites obtenidos de otros vegetales (Abobatta, 2019). Los reportes a nivel mundial 
destacan el aceite de JC como materia prima para la producción de biodiésel, por sus características fisicoquímicas (Martínez-Díaz et al., 2017; Martiñón et al., 2018; Wani et al., 2012), que ha mostrado tener mejor constitución en su perfil lipídico del aceite, calificado como oleico-linoleico con predominancia de ácidos grasos insaturados (monoinsaturados y poliinsaturados), constitución que le confiere al biodiésel un mejor desempeño en propiedades de flujo en frío (Campuzano-Duque \& Cardeño-López, 2020). En condiciones de Colombia, JC mostró un desempeño productivo con un costo de US $\$ 1,07$ (Caribe seco) y US \$1,24 (Valle interandino), en comparación con el costo del aceite de palma africana de US $\$ 0,89$ (Campuzano-Duque et al., 2020).

Los altos contenidos de ácido oleico y linoleico encontrados en los genotipos colombianos (CampuzanoDuque \& Cardeño-Lopez, 2020), están asociados con un alto índice de cetano y buena estabilidad oxidativa del biodiésel, dos características indicadoras de la calidad del producto (Nascimento et al., 2015). La acidez está considerada como una de las propiedades químicas más importante de un aceite y su valor está asociado con el proceso de transesterificación (Azees et al., 2019); valores bajos, menores al $1 \%$ como es el caso del aceite de los genotipos de JC estudiados en Colombia, son considerados óptimos para una transesterificación directa, a diferencia de los valores altos que con frecuencia son reportados en otros estudios (superior al $4 \%$ ), esta cualidad hace que el biodiésel obtenido pueda ocasionar deterioro a los elementos metálicos del motor y provoca corrosión, oxidación, decapado y daños a los inyectores (García-Muentes et al., 2018; Okullo \& Noah, 2017); por esta razón, el aceite requiere de una transesterificación ácida para disminuir el porcentaje de ácidos libres, seguida de una transesterificación alcalina (García-Muentes et al., 2018; Verma et al., 2019).

En Colombia, aun no se han caracterizado las propiedades químicas del aceite de JC en los genotipos de la colección Jatropha Colombia. Por ello, se realizó esta investigación con el objetivo de identificar en el aceite de cinco genotipos de JC en Colombia los índices de cetano, peróxido y yodo, el valor de acidez, el poder calorífico y verificar si cumplen con las normas internacionales para producción de biodiésel.

\section{Materiales y métodos}

\section{Localización}

El experimento en campo fue realizado en el Centro de Investigación Nataima, de la Corporación Colombiana de Investigación Agropecuaria (AGROSAVIA), ubicado en el municipio del Espinal, departamento del Tolima, Colombia, con una localización de $04^{\circ} 09^{\prime}$ latitud norte y $74^{\circ} 53^{\prime}$ longitud oeste. Se encuentra a una altura de 323 msnm, con una temperatura promedio de $29^{\circ} \mathrm{C}$, precipitación anual de $1380 \mathrm{~mm}$, humedad relativa promedio de $74 \%$.

\section{Diseño experimental}

Se utilizó un diseño de bloques completos al azar con cinco tratamientos y tres repeticiones. Los tratamientos se constituyeron por cinco genotipos denominados CJC3, CJC4, CJC7, CJC8, CJC9, que hacen parte de la Colección Jatropha Colombia, en custodia de AGROSAVIA, conformada por cuatrocientos accesiones colectadas por AGROSAVIA en el año 2008. Estos genotipos fueron seleccionados durante la cosecha del año 2014 por su alto rendimiento (Campuzano-Duque et al., 2020). La unidad experimental se constituyó de veinte plantas, establecidas a una distancia de siembra de $3 \times 2 \mathrm{~m}$.

Agron. Mesoam. 33(1): Artículo 44555, 2022

ISSN 2215-3608 doi:10.15517/am.v33i1.44555 


\section{Preparación de la muestra}

La cosecha se realizó en el año 2015, a los 36 meses de establecido el cultivo, en 20 plantas y 50 frutos por planta con coloración amarilla-café, de acuerdo con el índice de color del epicarpio (Campuzano \& Cardeño, 2017). De forma manual, se realizó la separación de los componentes del fruto en: cáscara, semilla y almendra. De la almendra se obtuvo el aceite por el método Soxhlet (Jensen, 2007), descrito así: se pesaron entre 8 a $10 \mathrm{~g}$ de la semilla molida de Jatropha, los cuales se llevaron a un cartucho soxhlet con su respectivo montaje que tenía hexano como solvente de extracción; el sistema se llevó hasta una temperatura de $70{ }^{\circ} \mathrm{C}$ y se dejó durante $12 \mathrm{~h}$. Se verificó durante todo el tiempo la recirculación continua del solvente y, de esta manera, garantizar que todo el aceite fuera extraído. Una vez terminado el tiempo de extracción definido, se desmontó el sistema soxhlet y se separó el extracto, mediante destilación al vacío en un rotoevaporador. En este proceso se recuperó todo el solvente (que puede ser empleado en otro proceso de extracción).

\section{Determinación de ácidos grasos y propiedades químicas de aceite}

Se determinaron cinco propiedades en el aceite: a) índice de cetano: se utilizó la ecuación de regresión con un ajuste del modelo $\mathrm{R}^{2}=0,953$ (Sánchez-Borroto et al., 2012) a partir del perfil de cinco ácidos grasos: CI (índice de cetano) $=0,342$ (linoleico) $+0,427$ (linolénico) + 0,561 (oleico) + 0,742 (palmítico) + 0,858 (esteárico) y expresado en cetanos. Los ácidos grasos se determinaron por cromatografía GC-MS y CG-FID en los cinco genotipos de JC para los ácidos grasos oleico (monoinsaturado), linoleico y linolénico (poliinsaturado), palmítico y esteárico (saturados); b) valor de acidez: calculado con base en los establecido por ASTM D974, proceso que implicó el uso de un estándar volumétrico de $\mathrm{KOH}$, junto con un disolvente de valoración y una solución indicadora (ASTM D974) para determinar la cantidad total, medida en mg de $\mathrm{KOH}$ que fueron necesarios para neutralizar los ácidos libres presentes en $1 \mathrm{~g}$ de aceite; c) índice de peróxido: determinado por valoración, tal como se describe en Ekop et al. (2007), se expresó como los miliequivalentes (meq) de peróxido por kg de aceite; d) índice de yodo: determinado por el método 993,20 AOAC (Association of Oficcial Analytical Chemists, 1990), calculado con la fórmula índice yodo $(\mathrm{IY})=(\mathrm{VB}-\mathrm{VM}) \times \mathrm{N}$ (Tiosulfato) $\times 0,127 \mathrm{~g} \mathrm{meq}^{-1} \times 100$, donde $\mathrm{VB}=$ volumen de tiosulfato de sodio gastado en la valoración de blanco y $\mathrm{VM}=$ volumen de tiosulfato de sodio gastado en la valoración de la muestra, el resultado se expresó en $\mathrm{mg} \mathrm{g}^{-1} \mathrm{y}$, e) poder calorífico: determinado con el uso de un calorímetro (bomba oxígeno IKA200; 30 bar y 97,7 \% oxígeno) y la aplicación del procedimiento descrito en ASTM E711-87.

\section{Análisis estadístico}

Con los datos de las variables determinadas se verificaron los supuestos teóricos del análisis de varianza: 1) la prueba de Shapiro \& Wilk (1965), para la homogeneidad de varianza, 2) análisis individual de varianza y 3) comparación de medias, que se realizó con la prueba de Tukey $(\mathrm{p}=0,01)$. Los procedimientos y análisis estadísticos se realizaron con el programa SAS versión 9.4 (Statistical Analysis Systems Institute, 2016).

\section{Resultados}

El factor genotipo en las variables ácidos grasos oleico, linoleico, linolénico y palmítico, presentaron diferencias significativas $(\alpha<0,05)$ y no significativas para esteárico y diferencias significativas para los parámetros de índices de cetano, peróxido y yodo, acidez y poder calorífico. Los coeficientes de variación en todos los casos no superaron el $10 \%$, lo cual indicó buen grado de confiabilidad de los datos.

Agron. Mesoam. 33(1): Artículo 44555, 2022 ISSN 2215-3608 doi:10.15517/am.v33i1.44555 
La participación de ácidos grasos en el aceite de los genotipos de JC fue mayor en los monoinsaturados (oleico) y poliinsaturados (linoleico). Los valores de ácido oleico variaron entre 47,93\% (CJC3) y 40,38\% (CJC9); mientras que los del ácido linoleico estuvieron entre 35,89\% (CJC3) y 28,91\% (CJC9), en ambos casos diferentes estadísticamente $(\alpha<0,05)$. En menor proporción se obtuvieron los ácidos palmítico y esteárico (saturados) y linolénico (poliinsaturado); en el caso del palmítico los valores variaron entre 13,44\% (CJC3) y 11,19\% (CJC9), el esteárico varió entre 8,26 \% (CJC3) y 7,05\% (CJC9) y el linolénico estuvo entre 0,26 \% (CJC3) y 0,17 \% (CJC9), diferente estadísticamente $(\alpha<0,05)$. CJC9 fue el genotipo que presentó los menores valores para todos los ácidos grasos determinados (Cuadro 1).

Cuadro 1. Valores promedio de ácidos oleico, linoleico, linolénico, palmítico y esteárico del aceite de cinco genotipos de Jatropha curcas. Corporación Colombiana de Investigación Agropecuaria (AGROSAVIA). Espinal, Colombia. 2015.

Table 1. Average values of oleic, linoleic, linolenic, palmitic, and stearic acids in oil of five Jatropha curcas genotypes. Corporacion Colombiana de Investigacion Agropecuaria (AGROSAVIA). Espinal, Colombia. 2015.

\begin{tabular}{cccccc}
\hline Genotipo & Oleico \% & Linoleico \% & Linolénico \% & Palmítico \% & Esteárico \% \\
\hline CJC3 & $47,93 \mathrm{a}$ & $35,89 \mathrm{a}$ & $0,26 \mathrm{a}$ & $13,44 \mathrm{a}$ & $8,26 \mathrm{a}$ \\
CJC4 & $47,93 \mathrm{a}$ & $34,29 \mathrm{ab}$ & $0,21 \mathrm{~b}$ & $12,65 \mathrm{a}$ & $7,83 \mathrm{a}$ \\
CJC7 & $44,26 \mathrm{ab}$ & $30,25 \mathrm{ab}$ & $0,22 \mathrm{~b}$ & $12,97 \mathrm{a}$ & $7,33 \mathrm{a}$ \\
CJC8 & $41,46 \mathrm{ab}$ & $31,49 \mathrm{ab}$ & $0,19 \mathrm{~b}$ & $12,47 \mathrm{ab}$ & $7,72 \mathrm{a}$ \\
CJC9 & $40,38 \mathrm{~b}$ & $28,91 \mathrm{c}$ & $0,17 \mathrm{~b}$ & $11,19 \mathrm{~b}$ & $7,05 \mathrm{a}$ \\
\hline
\end{tabular}

a,b,c Medias con diferente letra en la misma columna indican diferencias significativas ( $\mathrm{p}=0,05$, Tukey). / ${ }^{\mathrm{a}, \mathrm{b}, \mathrm{c}}$ Means with different letter in the same column indicate significant differences ( $\mathrm{p}-0.05$, Tukey).

El índice de cetano como índice relativo de la mayor o menor inflamabilidad de un biocombustible para motores de combustión interna (tipo diésel), presentó los valores más altos e iguales estadísticamente entre sí, en los genotipos CJC3 $(56,34)$, CJC4 $(54,81)$ y CJC7 $(51,18)$ y los de menor valor con los genotipos CJC8 $(46,97)$ y CJC9 $(49,99)$, sin diferir estos dos últimos estadísticamente. El valor de acidez presentó valores de $0,72 \mathrm{mgKOH} \mathrm{g}^{-1}$ para el genotipo CJC3 y 0,52 $\mathrm{mgKOH} \mathrm{g}^{-1}$ para el genotipo CJC9, diferentes estadísticamente ( $\left.\mathrm{p}=0,05\right)$ (Cuadro 2).

Cuadro 2. Comparación de las medias de los índices de cetano, peróxido y yodo, acidez, y poder calorífico del aceite de cinco genotipos de Jatropha curcas. Corporación Colombiana de Investigación Agropecuaria (AGROSAVIA). Espinal, Colombia. 2015.

Table 2. Average comparison of cetane, peroxide and iodine indexes, acidity, and calorific power of the five Jatropha curcas genotypes oil. Corporacion Colombiana de Investigacion Agropecuaria (AGROSAVIA). Espinal, Colombia. 2015.

\begin{tabular}{|c|c|c|c|c|c|}
\hline \multirow[t]{2}{*}{ Genotipo } & \multirow[t]{2}{*}{ Índice de cetano } & \multicolumn{3}{|c|}{ Índice } & \multirow{2}{*}{$\begin{array}{l}\text { Poder calorífico } \\
\qquad \mathrm{J} \mathrm{g}^{-1}\end{array}$} \\
\hline & & Acidez mg KOH g-1 & Peróxido meq $\mathrm{kg}^{-1}$ & Yodo $\mathrm{mg} \mathrm{g}^{-1}$ & \\
\hline CJC3 & $56,34 \mathrm{a}$ & $0,72 \mathrm{a}$ & $3,66 \mathrm{a}$ & $97,25 \mathrm{a}$ & $46312 \mathrm{a}$ \\
\hline CJC4 & $54,81 \mathrm{a}$ & $0,68 \mathrm{a}$ & $3,39 \mathrm{a}$ & $93,55 \mathrm{ab}$ & $47117 \mathrm{a}$ \\
\hline CJC7 & $51,18 \mathrm{a}$ & $0,62 \mathrm{ab}$ & $3,00 \mathrm{~b}$ & $91,10 \mathrm{bc}$ & 44529 b \\
\hline CJC8 & $46,97 \mathrm{~b}$ & $0,58 \mathrm{~b}$ & $3,11 \mathrm{~b}$ & $90,21 \mathrm{bc}$ & $41353 \mathrm{~b}$ \\
\hline CJC9 & $49,99 \mathrm{~b}$ & $0,54 \mathrm{~b}$ & $2,81 \mathrm{~b}$ & $87,97 \mathrm{~d}$ & $40853 \mathrm{~b}$ \\
\hline ASTM D6751 & $47 \mathrm{~min}$. & $0,5 \max$ & - & $130 \max$. & - \\
\hline EN 14214 & $51 \mathrm{~min}$. & $0,5 \max$. & - & $120 \max$. & - \\
\hline
\end{tabular}

Medias con diferente letra en la misma columna son diferentes estadísticamente ( $\mathrm{p}=0,05$, Tukey) / Means with different letter in the same column are statistically different (p-0.05, Tukey).

Agron. Mesoam. 33(1): Artículo 44555, 2022

ISSN 2215-3608 doi:10.15517/am.v33i1.44555 
El índice de peróxido presentó valores que variaron entre 3,66 meq kg-1 (CJC3) a 2,81 meq kg-1 (CJC9), los cuales fueron diferentes estadísticamente $(\alpha<0,05$; Cuadro 2$)$.

El índice de yodo, como una medida de los ácidos grasos insaturados, presentó el mayor valor con el genotipo CJC3 con 97,25 mg g ${ }^{-1}$ y el menor valor $87,97 \mathrm{mg} \mathrm{g}^{-1}$ con el genotipo CJC9 (Cuadro 2). El poder calorífico presentó el mayor valor con el genotipo CJC4 con $47117 \mathrm{~J} \mathrm{~g}^{-1} \mathrm{y}$ el menor con el genotipo CJC9 con $40853 \mathrm{~J} \mathrm{~g}^{-1}$, ambos resultados diferentes estadísticamente ( $\mathrm{p}=0,05$; Cuadro 2$)$.

El aceite de los genotipos de JC en este estudio presentó tres veces más de contenido de ácido linoleico, casi el doble del ácido esteárico y tres veces menos de ácido palmítico en comparación con el aceite de palma; esta constitución lipídica del aceite de los genotipos de JC estudiados mostró una superioridad de 1,4 veces más del índice de cetano, dos veces más del valor de acidez y 1,7 veces más en el índice de peróxido y yodo, en comparación con el aceite de palma; el poder calorífico del aceite de JC fue superior al aceite de palma de aceite (Cuadro 3).

Cuadro 3. Comparación del perfil lípidico y propiedades químicas de Jatropha curcas y Elaeis guineensis (Palma de aceite). Corporación Colombiana de Investigación Agropecuaria (AGROSAVIA). Espinal, Colombia. 2015.

Table 3. Comparison of the lipidic profile and chemical properties of Jatropha curcas and Elaeis guineensis (Oil Palm). Corporación Colombiana de Investigación Agropecuaria (AGROSAVIA). Espinal, Colombia. 2015.

\begin{tabular}{|c|c|c|c|c|c|c|c|c|c|}
\hline & \multirow[b]{3}{*}{$\begin{array}{c}\text { Oléico } \\
\%\end{array}$} & \multirow[b]{3}{*}{$\begin{array}{c}\text { Linoléico } \\
\%\end{array}$} & \multirow[b]{3}{*}{$\begin{array}{c}\text { Palmítico } \\
\%\end{array}$} & \multirow[b]{3}{*}{$\begin{array}{c}\text { Esteárico } \\
\%\end{array}$} & \multirow[b]{3}{*}{$\begin{array}{c}\text { Número } \\
\text { cetano }\end{array}$} & \multicolumn{3}{|c|}{ Índice } & \multirow[b]{3}{*}{$\begin{array}{c}\text { Poder calorífico } \\
\text { MJ g }^{-1}\end{array}$} \\
\hline & & & & & & Acidez & Peróxido & Yodo & \\
\hline & & & & & & $\begin{array}{c}\text { mg KOH } \\
\mathrm{g}^{-1}\end{array}$ & $\mathrm{mEq} \mathrm{kg}^{-1}$ & $\mathrm{mg} \mathrm{g}^{-1}$ & \\
\hline $\begin{array}{l}\text { Palma } \\
\text { africana }^{1}\end{array}$ & $36-44$ & $9-12$ & $39-47$ & $3-6$ & $35-39$ & $0,27-0,32$ & $2,28-2-45$ & $50-55$ & $37-39$ \\
\hline $\begin{array}{l}\text { Jatropha } \\
\text { curcas }^{2}\end{array}$ & $40-47$ & $28-35$ & $11-13$ & $7-8$ & $50-56$ & $0,54-0,72$ & $2,81-3,66$ & $87-97$ & $41-47$ \\
\hline
\end{tabular}

${ }^{1}$ Fuente: Rincón \& Martínez, 2009; ${ }^{2}$ Fuente: Campuzano \& Cardeño (resultados del estudio 2015). $/{ }^{1}$ Source: Rincón \& Martínez, 2009; ${ }^{2}$ Source: Campuzano \& Cardeño, 2015 (results of the study).

\section{Discusión}

Los valores de los ácidos grasos en el aceite de los genotipos estudiados, coincidieron con los perfiles lipídico del aceite de JC reportados a nivel mundial (Akbar et al., 2009; Falasca \& Ulberich, 2008; Martiñón et al., 2018; Salih \& Yahia, 2019; Verma et al., 2019), lo cual indicó que es un aceite con predominancia de ácidos grasos monoinsaturados (oleico C18:1) y poliinsaturados (linoleico C18:2), perfil lipídico que le confiere propiedades químicas al aceite para mayor estabilidad en la oxidación y menor punto de fusión, lo que podría contribuir a que el biodiésel elaborado presente mejor comportamiento de flujo en ambientes fríos (Sarin et al., 2007; Verma et al., 2019).

En relación con el índice de cetano, en Jatropha curcas, por su naturaleza aún silvestre, en proceso de mejoramiento genético (Campuzano et al., 2016), los valores reportados en otras investigaciones presentaron valores amplios entre 38,0 y 51,0 cetanos con una media de 46,3 cetanos (Achten et al., 2008). No obstante, los resultados encontrados en este estudio para este parámetro fueron cercanos con la media de 46,3 cetanos reportada por Achten et al. (2008) y similares a los reportados por otros investigadores de 51,0 (Xuan Ho et al., 2020), 57,1 (Sarin et al., 2007); 58,4 (Knothe \& Steidely, 2005); 57,4 (Sarin et al., 2007) y 54,2 a 56,5 (Verma et al., 2019). Contrario a lo anterior, con resultados inferiores a lo encontrado en Colombia, se encontraron reportes por Rodríguez-Acosta et al. (2010) de 43,0 cetanos y por Azeez et al. (2019) de 41,0 cetanos. El aceite de JC en Colombia ha mostrado valores de 
este índice que están dentro del rango para el funcionamiento apropiado del aceite en motores diésel y cumplen con los estándares internacionales de 47-65 (ASTM D6751) o 51 (EN 14214) (Knothe \& Steidely, 2005). No obstante, los motores diésel lentos exigen combustibles con índice de cetano comprendido entre 30 y 50, mientras que los diéseles rápidos los necesitan de 55-70.

La acidez del aceite de JC es una propiedad química que presenta una alta variación con una amplitud de 0,92 a $6,16 \mathrm{mg} \mathrm{KOH} \mathrm{g}^{-1}$ y una media de 3,0 $\mathrm{mg} \mathrm{KOH} \mathrm{g}^{-1}$ (Rodríguez-Acosta et al., 2010). En este trabajo, el aceite de los genotipos de JC estudiados los valores de índice de acidez variaron entre 0,54 y $0,72 \mathrm{mg} \mathrm{KOH} \mathrm{g}^{-1}$, datos que fueron menores a los valores reportados por otros estudios en el aceite de JC con 2,73 $\mathrm{mg} \mathrm{KOH} \mathrm{g}^{-1}$ (García-Muentes et al., 2018), 4,24 mg KOH g-1 (Adebowale \& Adedire, 2006), 4,56 mg KOH g ${ }^{-1}$ (Azeez et al., 2019; Ikyenge et al., 2019), $12,78 \mathrm{mg} \mathrm{KOH} \mathrm{g}^{-1}$ (Ong et al., 2013) y $22,76 \mathrm{mg} \mathrm{KOH} \mathrm{g}^{-1}$ (Mehmood et al., 2019) y un caso atípico con un valor significativamente alto de $27,78 \mathrm{mg} \mathrm{KOH} \mathrm{g}^{-1}$ (Aigba et al., 2021). En todos los casos, el índice de acidez del aceite de los cinco genotipos estudiados estuvo cercano a los límites permisibles en las normas ASTM D6751 y EN 14214 para biodiésel (Sanjid et al., 2013). Los valores hallados de acidez pueden ser aceptados para incluir el aceite como materia prima para la producción de biodiésel con catálisis alcalina, puesto que no se requieren pretratamientos.

Los valores obtenidos de índice de yodo expresado en $\mathrm{mg} \mathrm{g}^{-1}$, estuvieron dentro del rango reportado por Atchen et al. (2008) de 92-112 con una media de 101. Asimismo, estos tuvieron similitud con los valores reportados de 93,0 (Azam et al., 2005), 96,0 (Naresh et al., 2012), 82,0-98,0 (Belagur \& Chitimi, 2013), 90,0 (Crane et al., 2005) y 99,20 (Azeez et al., 2019), pero fueron diferentes a otros trabajos que mostraron resultados de 75,2 (Ansari et al., 2011), 71.5 (Azam et al., 2005), 106,1 (Ikyenge et al., 2019), 111,6 (Adebowale \& Adedire, 2006), 103,0 (Marutani et al., 2018), 110,0 (Ita et al., 2018), hasta valores más disímiles de 177,63 (Mehmood et al., 2019) y 216,2 (Akminul et al., 2013). Cuando el índice de yodo es bajo (26 a 48), el aceite está saturado y tiende a solidificarse, pero si aumenta de 94 a 135, el nivel de insaturaciones aumenta en las cadenas carbono, el aceite permanece líquido y esto repercute en la viscosidad. Los valores encontrados en este estudio fueron menores al promedio reportado para JC de 101 (Achten et al., 2008) e indican el grado de insaturación, potencial oxidativo y estabilidad del aceite y/o biodiésel; por lo tanto, es indicativo de que el aceite de los cinco genotipos estudiados tuvo calidad apropiada con base en la especificación europea de biodiésel (EN 14214) que limita el valor máximo de yodo a 120 (Atadashi et al., 2010; Ong et al., 2011).

El índice de peróxido ha mostrado valores en meq $\mathrm{kg}^{-1}$ desde 0,30 (Ita et al., 2018), 3,5 (Adebowale \& Adedire, 2006), hasta 11,50 (Mehmood et al., 2019). Los cinco genotipos estudiados en el presente trabajo, presentaron resultados para este parámetro menores a 5,0, considerado apropiado, ya que valores superiores a 5,0 pueden repercutir en la formación de polímeros de alto peso molecular (gomas), que podrían ocasionar problemas en el funcionamiento del motor.

En JC se reportó un valor de poder calorífico expresado en $\mathrm{J} \mathrm{g}^{-1}$ que varió entre 37830 a 42 050, con una media de 39630 (Achten et al., 2008). Los resultados obtenidos en la presente investigación, concordaron con los reportes de 38200 a 39660 (Augustus et al., 2002; Pramanik, 2003), 39250 (Sahoo \& Das, 2009), 38500 (Sahoo \& Das, 2009), 39230 (Ong et al., 2011), 41397 (Ong et al., 2011), 39100 a 43000 (Belagur \& Chitimi, 2013), 39730 (Xuan Ho et al., 2020) 42000 a 45900 (Sanjid et al., 2013), 39000 a 41000 (Oliveira \& Da-Silva, 2013), 38500 a 40100 (Nwokocha \& Adegbuyiro, 2017) y 38240 (Jonas et al., 2018). Contrario a lo anterior, se conoce de un reporte donde el poder calorífico del aceite de JC fue menor con un valor de $19280 \mathrm{~J} \mathrm{~g}^{-1}$ (Kethobile et al., 2020). Sobresalió, el poder calorífico del genotipo CJC4 de 47 117, superior al del diésel y keroseno de 45 843, carbón de 33 000, madera de 22000 y soya-colza de 39500 (Oliveira \& Da-Silva, 2013).

Las propiedades químicas favorables del aceite de JC, además de su constitución lipídica oleico-linoleico, el biodiésel derivado del aceite de los genotipos estudiados tuvo buena estabilidad oxidativa (Verma et al., 2019) y menor punto de fusión y mejor desempeño en frío, así como mayor poder de ignición del biodiésel (Knothe, 2009), en comparación con aceites como el de palma.

Agron. Mesoam. 33(1): Artículo 44555, 2022

ISSN 2215-3608 doi:10.15517/am.v33i1.44555 


\section{Conclusiones}

El aceite de los cinco genotipos de JC evaluados en Colombia, presentaron dominancia de los ácidos grasos monoinsaturados (oleico C18:1) y poliinsaturados (linoleico C18:2). Sobresalió el bajo índice de acidez de todos los genotipos, que lo hace adecuado para el proceso de producción de biodiésel sin requerir pretratamientos.

El aceite de todos los genotipos estudiados cumplió con los parámetros de cetano y yodo requeridos por las normas ASTM y EN para el biodiésel, razón por la cual se consideran apropiados para la elaboración de este biocombustible.

\section{Agradecimientos}

La información usada para este trabajo fue resultado del Contrato RC. No. 786 -2011 entre la Fiduciaria de Bogotá como vocera del patrimonio autónomo denominado Fondo Nacional de Financiamiento para la Ciencia, la Tecnología y la Innovación Francisco José de Caldas, Corporación Colombiana de Investigación Agropecuaria(AGROSAVIA), la Universidad de Antioquia - Universidad de Antioquia (DEA) y Carbones Cerrejón Limited.

\section{Referencias}

Adebowale, K. \& Adedire, C. O. (2006). Chemical composition and insecticidal properties of the underutilized Jatropha curcas seed oil. African Journal of Biotechnology, 5(10), 901-906. http://www.academicjournals.org/AJB

Abobatta, W. (2019). Jatropha curcas: an overview. Journal of Advances in Agriculture, 10, 1650-1656. https://doi. org/10.24297/jaa.v10i0.8145

Achten, W. M., Verchot, L., Franken, Y. J., Mathijs, E., Singh, V. P., Aerts, R., \& Muys, B. (2008). Jatropha bio-diesel production and use. Biomass and Bioenergy, 32(12), 1063-1084. https://doi.org/10.1016/j.biombioe.2008.03.003

Aigba, P. I, Anyadiegwu, F. C., \& Ogoke, J. C (2021). Characterization of Jatropha Oil and its Biodiesel. Advance Environmental Studies, 5(1), 376-381.

Akbar, E., Yaacob, Z., Kamaudin, S., Ismail, M., \& Salimon, J. (2009). Characteristic and composition of Jatropha curcas oil seed from Malaysia and its potential as biodiesel feedstock. European Journal of Scientific Research, 29(3), 396-403.

Akminul, A. K. M., Primandari, S. R. P., Yaakob, Z., Anuar, N., \& Osman, M. (2013). The properties of Jatropha curcas seed oil from seven different countries. Energy Sources, 35(18), 698-1703. https://doi.org/10.1080/15567036.2010.529572

Aminul, K. M., Yaakob, Z., Anuar, N., Primandari, P., \& Osmand. M. (2012). Physicochemical properties of Jatropha curcas seed oil from different origins a candidate plus plants (CPPs). Journal of the American Oil Chemicals Society, 89(2), 293-300. https://doi.org/10.1007/s11746-011-1908-7

Ansari S. H., Naseem, M., Hasnat, A., \& Ahmad S. A. (2011). Polyesteramide resin from Jatropha curcas seed oil for anticorrosive coating. Biosciences Biotechnology Research Asia, 8(2), 1-16. https://doi.org/10.13005/bbra/944

Association of Official Analytical Chemists. (1990). Official methods of analysis (15 ${ }^{\text {th }}$ Ed.). AOAC Int.

Atadashi, I. M., Aroua, M. K., \& Aziz, A. A. (2010). High quality biodiesel and its diesel engine application. A review. Renewable Sustainable Energy Reviews, 14(7), 1999-2008. https://doi.org/10.1016/j.rser.2010.03.020

Agron. Mesoam. 33(1): Artículo 44555, 2022 ISSN 2215-3608 doi:10.15517/am.v33i1.44555 
Augustus, G. D. P., Jayabalan, M., Seiler, G. J. (2002) "Evaluation and bioinduction of energy components of Jatropha curcas. Biomass and Bioenergy, 23, 161-164. https://doi.org/10.1016/S0961-9534(02)00044-2

Azam, M.,Amtul, M., \& Nahar, N. M. (2005). Prospects and potential of fatty acid methyl esters of some non-traditional seed oils for use as biodiesel in India. Biomass and Bioenergy, 29(4), 293-302. https://doi.org/10.1016/j.biombioe.2005.05.001

Azeez, A. M., Fasakin, A. O., \& Orege, J. I. (2019). Production, characterization and fatty acid composition of Jatropha curcas biodiesel as a viable alternative to conventional diesel fuel in Nigeria. Green and Sustainable Chemistry, 9(1), 1-10. https://doi.org/10.4236/gsc.2019.91001

Belagur, V. K., \& Chitimi, V. R. (2013). Few physical, chemical and fuel related properties of Calophyllum inophyllum Linn (honne) oil and its blends with diesel fuel for their use in diesel engine. Fuel, 109, 356-361. https://doi.org/10.1016/j. fuel.2013.02.015

Benatti, P., Peluso, G., Nicolai, R., \& Calvani, M. (2004). Polyunsaturated fatty acids: biochemical, nutritional and epigenetic properties. Journal of the American College of Nutrition, 23(4), 281-302. https://doi.org/10.1080/07315724.2004.1 0719371

Campuzano, L. F., \& Cardeño, F. (2017). Measurement of fruit color-heterogeneity index and their relation to Jatropha curcas L., oil in Colombia. Acta Agronómica, 66(1), 9-14. https://doi.org/10.15446/acag.v66n1.50736

Campuzano, L. F., Ríos, L. A., \& Cardeño, F. (2016). Caracterización composicional del fruto de 15 variedades de Jatropha curcas L. en el departamento del Tolima, Colombia. Revista Ciencia \& Tecnología Agropecuaria, 17(3), 379-390. https://doi.org/10.21930/rcta.vol17_num3_art:514

Campuzano-Duque, L.F., \& Cardeño-Lopez, F. (2020). Composición de los ácidos grasos de genotipos de Jatropha curcas L., en Colombia. Agronomía Mesoamericana, 31(1), 95-104. https://doi.org/10.15517/am.v31i1.37574

Campuzano-Duque, L.F., Guadrón-Acosta, R., \& Chávez-Oliveros, L.F. (2020). Desempeño productivo y adaptación de Jatropha curcas L. en tres ecorregiones en Colombia. Agronomía Mesoamericana, 31(2), 277-290. https://doi. org/10.15517/am.v31i2.39213

Crane, S., Aurore, G., Joseph, H., Mouloungui, Z., \& Bourgeois, P. (2005). Composition of fatty acids triacylglycerols and unsaponifiable matter in Calophyllum calaba L. oil from Guadeloupe. Phytochemistry, 66(15), 1825-1831. https:// doi.org/10.1016/j.phytochem.2005.06.009

Ekop, S. A., Etuk, B. A., \& Eddy, N. O. (2007). Effect of some local additives on the chemical constituent of palm oil. Journal of Applied Sciences and Environmental Management, 11(1), 85-89. https://doi.org/10.4314/jasem.v11i1.46840

Falasca, S. L., \& Ulberich, A. (2008). Potencialidad bioenergética sudamericana a partir de forestaciones con Jatropha sp. (J. curcas, hieronymi y macrocarpa). Revista Virtual Redesma, 2(2), 101-116. http://www.revistasbolivianas.org.bo/ scielo.php?script=sci_arttext\&pid=S1995-10782008000200007\&lng=pt\&nrm=iso

García-Dávila, J., Ocarranza, E., Rojas, M., Muñoz, J., Ramírez, J., \& Martinez, A. (2014). Jatropha curcas L. oil hydroconversion over hydrodesulfurization catalysts for biofuel production. Fuel, 135(1), 380-386. https://doi. org/10.1016/j.fuel.2014.07.006

García-Muentes, S. A., Lafarque-Peréz, F., Labrada-Vásquez, B., Días-Velasquez, C. M., \& Campo-Lafita, A. E. (2018). Propiedades fisicoquímicas del aceite y biodiesel producidos de la Jatropha curcas L. en la provicia de Manabi, Ecuador. Revista Cubana de Química, 30(1), 142-158.

Agron. Mesoam. 33(1): Artículo 44555, 2022

ISSN 2215-3608 doi:10.15517/am.v33i1.44555 
Ikyenge, B. A., Agbidye, I. G., \& Ameh, S. O. (2019). Synthesis and characterization of poly ester amide urethane derived from Jathropha curcas seed oil from Nigeria. Journal of Chemical Society of Nigeria, 44(2), 257-262. http://journals. chemsociety.org.ng/index.php/jcsn/article/view/273

Ita, B. I., Magu, T. O., \& Ehi-Eromosele, C. O. (2018). Physico-chemical properties of biodiesel obtained from Jatropha curcas seeds oil using $\mathrm{CoMgFe}_{2} \mathrm{O}_{4}$ and $\mathrm{MgFe}_{2} \mathrm{O}_{4}$ as nanocatalysts. Journal of Industrial Technology, 3(1), 1-16.

Jain, S., \& Sharma, M. P. (2011). Oxidation stability of blends of jatropha biodiesel with diesel. Fuel, 90, 3014-3020. https:// doi.org/10.1016/j.fuel.2011.05.003

Jensen, W. B. (2007). The origin of the Soxhlet extractor. Journal of Chemical Education, 82, 1913-1914. https://doi. org/10.1021/ed084p1913

Jonas, M., Ketlogetswe, C., \& Gandure, J. (2018). Influence of Jatropha fruit maturity on seed oil yield, composition and heat of combustion of derived biodiesel. Energy and Power Engineering, 10(03), 77-86. https://doi.org/10.4236/ epe. 2018.103006

Kethobile, E., Ketlogetswe, C., \& Gandure, J. (2020). Characterisation of the non-oil Jatropha biomass material for use as a source of solid fuel. Biomass Conversion and Biorefinery, 10, 1251-1267. https://doi.org/10.1007/s13399-019-005114

Knothe, G. (2009). Improving biodiesel fuel properties by modifying fatty ester composition. Energy and Environmental Science, 2, 759-766. https://doi.org/10.1039/b903941d

Knothe, G., \& Steidley, K. R. (2005). Kinematic viscosity of biodiesel fuel components and related compounds. Influence of compound structure and comparison to petrodiesel fuel components. Fuel, 84(9), 1059-1065. https://doi. org/10.1016/j.fuel.2005.01.016

Martínez-Díaz, I., González-Rodríguez, A., Rico-Ponce, H. R., Rocha-Ramírez, V., Ovando-Medina, I., \& Espinosa-García, F. J. (2017). Fatty acid diversity is not associated with neutral genetic diversity in native populations of the biodiesel plant Jatropha curcas L. Chemistry \& Biodiversity, 14(1), Article e1600188. https://doi.org/10.1002/cbdv.201600188

Martiñón, A., Figueroa, R., Martínez, A., Martínez, J., Pacheco, G., \& García, J. (2018). Chemical and physical characterization of Jatropha curcas L., seed from the Northern of Puebla, México. Journal of Plant Sciences, 6(1), 25-30. https://doi. org/10.11648/j.jps.20180601.15

Marutani, M., Soria, J. A., \& Martinez, M. A. (2018). Characterization of crude and biodiesel oils of Jatropha curcas and Calophyllum inophyllum in Guam. Micronesica, 1, 1-15.

Mehmood, T., Naseem, A., Anwar, F., Iqbal, M., \& Shaheen, M. A. (2019). Jatropha curcas L.: A non-food oil source for optimized production. Journal of the Chemical Society of Pakistan, 41(3), 458.

Naresh, B., Reddy, M. S., Vijayalakshimi, P., Reddy, V., \& Devi, P. (2012). Physico-chemical screening of accessions of Jatropha curcas for biodiesel production. Chemistry Biomass \& Bioenergy, 40, 155-161. https://doi.org/10.1016/j. biombioe.2012.02.012

Nascimento, I.A., Cabanelas, I. T. D., dos-Santos, J. N., Nascimento, M. A., Sousa, L., \& Sansone, G. (2015). Biodiesel yields and fuel quality as criteria for algal-feedstock selection: effects of $\mathrm{CO}_{2}$-supplementation and nutrient levels in cultures. Algal Research, 8, 53-60. https://doi.org/10.1016/j.algal.2015.01.001 
Nwokocha, L. M., \& Adegbuyiro, A. (2017). Effect of roasting temperature on the physicochemical properties of Jatropha curcas Kernel oil extracted with cold hexane and hot water. African Journal of Pure and Applied Chemistry, 11(3), 19-29. https://doi.org/10.5897/AJPAC2017.0721

Okullo, A., \& Noah, T. (2017). Process Simulation of Biodiesel Production from Jatropha Curcas Seed Oil. American Journal of Chemical Engineering, 5(4), 56-63. https://doi.org/10.11648/j.ajche.20170504.12.

Oliveira L. E., \& Da Silva, M. L. (2013). Comparative study of calorific value of rapeseed, soybean, Jatropha curcas and Crambe biodiesel. Renewable Energy and Power Quality Journal, 1(1), 679-682. https://doi.org/10.24084/repqj11.411

Ong, H.C., Mahlia, T. M. I., Masjuki, H. H., \& Norhasyima, R. S. (2011). Comparison of palm oil, Jatropha curcas and Calophyllum inophyllum for biodiesel: A review. Renewable and Sustainable Energy Reviews, 15(8), 3501-3515. https://doi.org/10.1016/j.rser.2011.05.005

Ong, H. C., Silitonga, A. S., Masjuki, H. H., Mahlia, T. M. I., Chong, W. T., \& Boosroh, M. H. (2013). Production and comparative fuel properties of biodiesel from non-edible oils: Jatropha curcas, Sterculia foetida and Ceiba pentandra. Energy Conversion and Management, 73, 245-255. https://doi.org/10.1016/j.enconman.2013.04.011

Pramanik, K, (2003). Properties and use of Jatropha curcas oil and diesel fuel blends in compression ignition engine. Renewable Energy, 28(2), 239-248. https://doi.org/10.1016/S0960-1481(02)00027-7

Rincón, S. M., \& Martínez, D. M. (2009). Análisis de las propiedades del aceite de palma en el desarrollo de su industria. Revista Palmas, 30(2), 11-24. https://publicaciones.fedepalma.org/index.php/palmas/article/view/1432

Rodríguez-Acosta, M., Sandoval-Ramírez, J., \& Zeferino-Díaz, R. (2010). Extraction and characterization of oils from three Mexican Jatropha species. Journal Mexican Chemistry Society, 54(2), 88-91. https://doi.org/10.29356/jmcs.v54i2.950

Salih, M. N. K. M., \& Yahia, E. M. (2019). Phytochemical characterization of naturalized sudanese Jatropha curcas seed kernels. Asian Journal of Applied Chemistry Research, 3(4), 1-6. https://doi.org/10.9734/ajacr/2019/v3i430100

Sánchez-Borroto, Y., Piloto-Rodriguez, R., Goyos-Pérez, L., \& Ferrer-Frontela, N. (2012). Predicción del número de cetano de biocombustibles a partir de su composición de ácidos grasos. Ingeniería Mecánica, 15(2), 147-157.

Sarin R., M. Sharma, M., Sinharay, S., \& Malhotra, R. K. (2007). Jatropha-Palm biodiesel blends: An optimum mix for Asia. Fuel, 86(10-11), 1365-1371. https://doi.org/10.1016/j.fuel.2006.11.040

Sahoo, P. K., \& Das, L. M. (2009). Process optimization for biodiesel production from Jatropha, Karanja and Polanga oils. Fuel, 88(9), 1588-1594. https://doi.org/10.1016/j.fuel.2009.02.016

Sanjid, A., Masjuki, H. H., Kalam, M. A., Ashrafur Rahman, S. M., Abedin, M. J., \& Palash, S. M. (2013). Impact of palm, mustard, waste cooking oil and Calophyllum inophyllum biofuels on performance and emission of CI engine. Renewable and Sustainable Energy Reviews, 27, 664-682. https://doi.org/10.1016/j.rser.2013.07.059

Statistical Analysis Systems Institute. (2016). The SAS system for Windows (Release 9.4.). SAS Institute Inc.

Shapiro, S. S., \& Wilk, M. B. (1965). An analysis of variance test for normality (complete samples). Biometrika, 52(3-4), 591-611. https://doi.org/10.1093/biomet/52.3-4.591

Soliman, W. M., \& He, X. R. (2015). The potentials of Jatropha plantations in Egypt: A review. Modern Economy, 6(2), 190200. https://doi.org/10.4236/me.2015.62016

Agron. Mesoam. 33(1): Artículo 44555, 2022

ISSN 2215-3608 doi:10.15517/am.v33i1.44555 
Verma, R., Sharma, D. K., \& Bisen, P. S. (2019). Determination of free fatty acid composition in Jatropha crude oil and suitability as biodiesel feedstock. Current Alternative Energy, 3(1), 1-16. https://doi.org/10.2174/24054631036661 90722163037

Wani, T.A., Kitchulu, S., \& Ram, G. (2012). Genetic variability studies for morphological and qualitative attributes among Jatropha curcas L. accessions grown under subtropical conditions of North India. South African Journal of Botany, 79, 102-105. https://doi.org/10.1016/j.sajb.2011.10.009

Xuan Ho, N., Binh Pham, H., \& Nguyen Duy, V. (2020). Experimental study on characteristics of the test engine fueled by biodiesel-based Jatropha oil and traditional diesel. AIMS Energy, 8(6), 1143-1155. https://doi.org/10.3934/ energy.2020.6.1143

Zhu, L., Cheung, C. S., \& Huang, Z. (2016). Impact of chemical structure of individual fatty acid esters on combustion and emission characteristics of diesel engine. Energy, 107, 305-320. https://doi.org/10.1016/j.energy.2016.04.030 